\title{
Dielectric loss factor of sintered coal ash
}

\author{
Dorota Nowak-Woźny ${ }^{1, *}$, Leszek Woźny ${ }^{2}$, and Laura González Valdés ${ }^{1}$ \\ ${ }^{1}$ Faculty of Mechanical and Power Engineering, Wrocław University of Science and Technology, 27 \\ Wybrzeże Wyspiańskiego St, 50-370 Wrocław \\ ${ }^{2}$ Faculty of Electrical Engineering, Wrocław University of Science and Technology, 27 Wybrzeże \\ Wyspiańskiego St, 50-370 Wrocław
}

\begin{abstract}
This work presents the study of dissipation factor $(\tan \delta)$ for ash prepared from a power plant coal. Values of $\tan \delta$ were measured for samples of coal ash with additive of biomass and kaolinite, sintered at various temperatures up to $1100^{\circ} \mathrm{C}$. Procedure of direct $\tan \delta$ measurement is described and the results of loss factor as a function of frequency are shown. Quantitative and qualitative changes of $\tan \delta$ were observed for the different tested samples. The measurement data is compared with SEM microscopic images of the samples sintered at the maximum temperature. The results suggest that direct measurement of $\tan \delta$ can be a useful tool for slagging and fouling risk assessment.
\end{abstract}

\section{Introduction}

The massive built-up of ash deposits formed on heat exchange surfaces during coal, biomass, or other fuel combustion is a very important exploiting problem because it may force to interrupt the energy conversion process in order to clean the boiler's elements. Thus, it is needed to elaborate an assessing method able to predict with precision the slagging and/or fouling tendency of a fuel.

The slagging and fouling process occurs inside the boiler when ash particles collide with the boilers surface and heating tubes, condense on cooler elements and diffuse and react with the construction materials. Properties of such formed deposits depend on the ash temperature, the temperature of the surface on which the ash gathers, and on the fuel's composition.

Sintering phenomena are associated with the bonding or welding processes of the ash particles, which is closely associated with the physico-chemical processes that take place on the ash surface [1]. Because of the complex character of the sintering processes and of the complicated physico-chemical structure of the ash, it is difficult to transfer the real slagging and fouling process into laboratory conditions for precise prediction of slagging and fouling tendency. Deposits formation mechanisms on heating parts of boilers are connected with particular ash particles interactions. The interactions model for particular ash particles takes into account such properties as ash viscosity, ash grain size, ash grains surface tension and heat conduction of the surface at which the ash is gathered.

\footnotetext{
* Corresponding author: dorota.nowak-wozny@pwr.edu.pl
} 
The ash slagging and fouling process depends strongly on the surface tension and ash viscosity [2]. At high temperatures it appears a liquid phase on the surface of ash particles. For ash fraction of smaller grains the sintering effect starts for higher viscosity [3]. Viscosity and surface tension depend inversely on temperature. The mechanism of deposits formation is described in literature [2-10].

From physical point of view, the ash pellets used in standard tests, behave as ceramic material consisted of grains formed by different materials and with different dimensions and shapes. Consequently, the standard Ash Fusion Test (AFT) - widely used to assess slagging and fouling tendency in coal conversion process - may not represent accurately the sintering processes occurring at micro-scale of material and does not indicate exactly at which temperature the first melt/slag occurs. Contrarily, the first melt/slag point is very important in the real processes occurring in the boiler. The mechanical or pressure drop tests [11] seem to be more adequate because they are based on change of physical properties (mechanical strength and porosity) associated with the ash microstructure. Regarding to chemical reactions and phase transitions, tests based on phase equilibrium calculations [12-16] may be the way more appropriate.

Because of the dielectric properties of the ash, we suggest that the results from AC electrical test, especially dissipation factor versus frequency and sintering temperature, could be very interesting to analyse the problem.

The electrical properties of the ash depend on its microstructure and composition. The main components of the coal ash are aluminosilicates. In the ash it is common to encounter as well unburned coal particles. The existence of these particles may cause the change in electrical behaviour of ash attributed to the polarization effects on the grains surface or to ion conductivity depending on metal cations $[17,18]$. In ceramic materials dielectric losses are attributed to presence of electric dipoles and loosely bound ions as well as the space charge polarization related mainly to the granular microstructure of ash [19].

The complex chemical composition of the ashes, their structure, microstructure, and numerous heterogeneities can cause various internal dielectric losses. It has to be reflected in the values of various dielectric parameters and their characteristics depending on the frequency.

The dissipation factor $\tan \delta$ (also called the loss factor) is the parameter describing the internal friction for moving of coupling chargers (dipoles) and free charges (mainly ions) in dielectric materials. It can be used to determine the general dielectric properties of materials. Measurements of $\tan \delta$ can be the convenient and relatively simple method for quick diagnostics of various materials with high resistance, including ashes after combustion in electro-energetic power furnaces.

For these reasons, the presented work is an attempt to apply $\tan \delta$ measurement for characterization of various types of ash samples. The study does not include any other dielectric measurements. The aim was not to evaluate the dielectric properties of the materials but to assess the state of the ash by direct measurements of $\tan \delta$. Measurements were conducted for several samples of ash samples preheated at different temperatures $T_{\mathrm{s}}$. The loss factor measurements were analysed together with scanning electron microscopy (SEM) images.

\section{Samples}

The ash samples used in this work were obtained from three sort of fuels:

1. C1 hard coal ash,

2. S mixture of $80 \mathrm{wt} . \%$ of hard coal ash with $20 \mathrm{wt} . \%$ of cereal pellets ash,

3. S1 mixture of $95 \mathrm{wt} \% \%$ of hard coal ash with $5 \mathrm{wt} . \%$ of kaolinite.

The ashes were prepared according to the commonly applied procedure. 
The fuels were ground, sieved $(500 \mu \mathrm{m})$ and next heated in a muffle furnace at $500^{\circ} \mathrm{C}$ for 48 hours. Next, after re-grinding and mixing the obtained ash, samples were created in form of pellets ( $5 \mathrm{~mm}$ in diameter and about $4 \mathrm{~mm}$ in thickness) under a pressure of $1 \mathrm{MPa}$. Weigh percent of oxide content for tested samples is given in Table 1.

Table 1. Oxide content of samples, wt \%.

\begin{tabular}{|c|c|c|c|}
\hline Samples & C1 & S & S1 \\
\hline $\mathrm{SiO}_{2}$ & 48.9 & 47.7 & 51.2 \\
\hline $\mathrm{Fe}_{2} \mathrm{O}_{3}$ & 5.67 & 5.87 & 3.41 \\
\hline $\mathrm{Al}_{2} \mathrm{O}_{3}$ & 25.4 & 24.4 & 28.8 \\
\hline $\mathrm{Mn}_{3} \mathrm{O}_{4}$ & 1.06 & 1.00 & 0.91 \\
\hline $\mathrm{TiO}_{2}$ & 0.08 & 0.08 & 0.05 \\
\hline $\mathrm{CaO}$ & 3.65 & 3.65 & 2.24 \\
\hline $\mathrm{MgO}$ & 2.57 & 2.86 & 1.60 \\
\hline $\mathrm{SO} 3$ & 3.57 & 3.57 & 2.13 \\
\hline $\mathrm{P}_{2} \mathrm{O}_{5}$ & 0.60 & 2.28 & 0.59 \\
\hline $\mathrm{Na}_{2} \mathrm{O}$ & 0.98 & 0.94 & 0.64 \\
\hline $\mathrm{K}_{2} \mathrm{O}$ & 2.79 & 3.74 & 2.07 \\
\hline
\end{tabular}

\section{Measuring details}

Disssipation loss factor $\tan \delta$ is the convenient parameter for characterization of dielectric materials. It is defined as the power lost in the form of heat in the volume of the material. Its value depend on sample temperature and on frequency and intensity of external electric field.

It can be determined as

$$
\tan \delta=G /(\omega \varepsilon)
$$

where:

- $\quad G$ - dielectric bulk conductivity,

- $\omega$ - pulsation of the external electric field,

- $\varepsilon$ - permittivity of the dielectric.

Tested ash samples were sintered initially for 2 hours at fixed temperature of $500^{\circ} \mathrm{C}$. After sintering the samples were quenched to room temperature (by dropping to metal plate) in order to freeze the equilibrium state of the ash microstructure and phase composition. As quick as possible the annealed samples were electrically tested. Before each test surfaces of samples were polished for improvement electrical contact. The dissipation factor $(\tan \delta)$ measurements were carried out at room temperature for applied electrical field frequencies in range of $500 \mathrm{~Hz}$ to $1 \mathrm{MHz}$.

After electrical tests the samples were again sintered at 100 degree higher temperature and again quenched. This procedure was repeated up to $1100^{\circ} \mathrm{C}$. Such thermal treatment let us measure the dissipation factor in a thermodynamic equilibrium state. Repetition of sintering of the same sample at higher temperature and annealing up to a new equilibrium state give us the information about the sintering process step by step.

The dissipation factor was measured using QuadTech 7600 Plus Precision LCR Meter. The measured samples were placed between the brass electrodes in low humidity chamber. 
The measurements setup is presented in Figure 1. After overall annealed procedure the microstructure of samples was recorded using SEM COXEM EM-30AX.

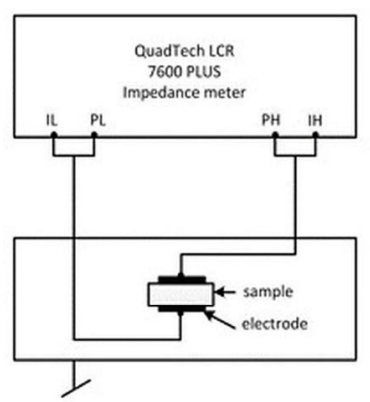

Fig. 1. Measurement setup.

\section{Dissipation factor measurements}

For samples $\mathrm{C} 1, \mathrm{~S}$ and $\mathrm{S} 1$ the dependences of $\tan \delta$ on the frequency for a fixed sintering temperature are presented on Figures 2, 3 and 4 respectively.

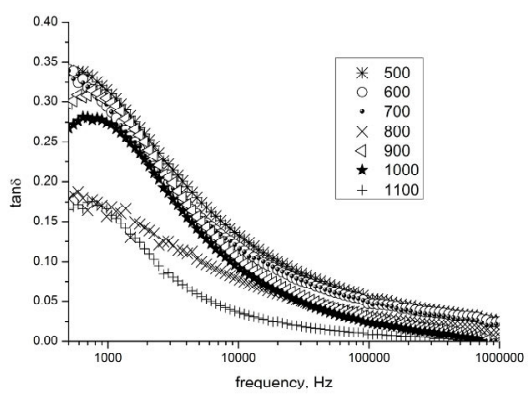

Fig. 2. Dependence of $\tan \delta$ on electric field frequency for sample $\mathrm{C} 1$ sintered at various temperatures.

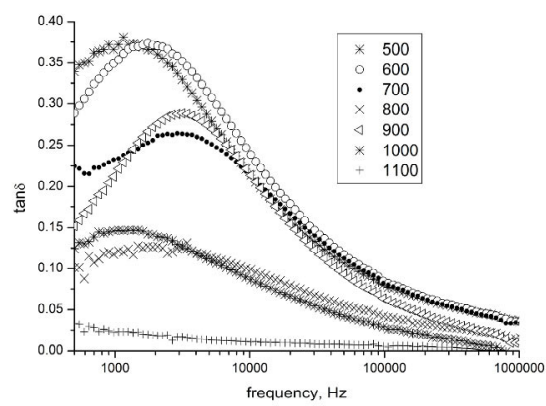

Fig. 3. Dependence of $\tan \delta$ on electric field frequency for sample S sintered at various temperatures. 


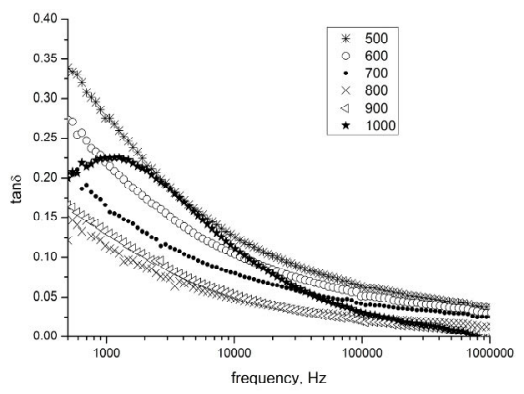

Fig. 4. Dependence of $\tan \delta$ on electric field frequency for sample S1 sintered at various temperatures.

As it can be seen in Figure 2 (sample $\mathrm{C} 1$ - pure coal ash) the plots show a peaking behaviour for all sintering temperatures. The tangent loss characterized by the appearance of the peak suggests the presence of relaxing dipoles in the specimen [20]. It was observed a soft shifting of the peaks towards higher frequencies and a decrease of the value of dissipation factor for higher temperatures. These effects exist and are visible in the graphs until $800^{\circ} \mathrm{C}$. At $900^{\circ} \mathrm{C}$ the $\tan \delta$ peak significantly increase and for samples sintered at higher temperatures - decreases and slightly shifts to lower frequencies. In all sintering temperature region the relaxation mechanism of energy dissipation is dominant. This suggest that $\tan \delta$ plot describes the same mechanism at various temperatures [21]. The frequency for maximum value of $\tan \delta$ changes in the range of $500 \mathrm{~Hz}$ to $1 \mathrm{kHz}$ and maximum dissipation factor value ranges from 0.38 to about 0.19 .

For sample S (ash of coal and biomass, Figure 3) the character of losses is similar as for pure coal ash (sample $\mathrm{C} 1$ ) but observed changes are more drastic. Dependence of $\tan \delta$ on frequency for sample sintered at $1100^{\circ} \mathrm{C}$ has different character suggesting a change of main power loss mechanism, which may be induced by a higher degree of crystallinity at that temperature [20]. Thus, the addition of biomass does not change the conduction mechanism when the samples are sintered at temperatures up to $1000^{\circ} \mathrm{C}$. Sintering at $1100^{\circ} \mathrm{C}$ involves a change in the conduction mechanism, probably associated with the slag phase formation.

For the third sample S1 (ash of coal and kaolinite, Figure 4) there is a more interesting issue. For samples sintered at temperatures in the range of 500 to $900^{\circ} \mathrm{C}$ all dependences have decreasing character. It suggests the existence of conductive dielectric loss mechanism as a dominant one. Values of $\tan \delta$ are less than 0.37 for all frequencies. But for the sample sintered at $1000^{\circ} \mathrm{C}$ a radical change in the nature of losses appears. Dependence of $\tan \delta$ on frequency has peaking character, suggesting the presence of relaxing dipoles [20]. This change in the trend of the graph may indicate a change of the dominant conduction mechanism which in turn, is temperature dependent.

The detailed explanation of dissipation factor changes for tested samples is very not easy and requires further research. 


\section{Microstructure observations}

The microstructure of the surface for all samples was studied under scanning electron microscope (SEM). The images are shown in Figures 5-7.

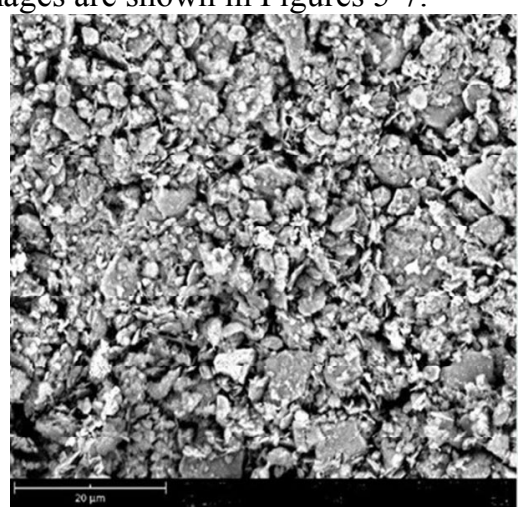

Fig. 5. Microstructure of sample $\mathrm{C} 1$ sintered at $1100^{\circ} \mathrm{C}$.

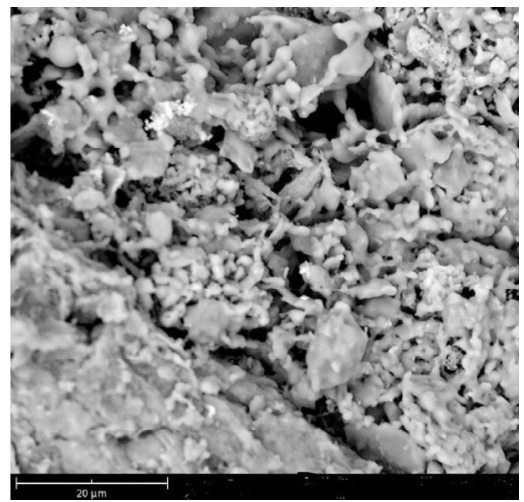

Fig. 6. Microstructure of sample S sintered at $1100^{\circ} \mathrm{C}$.

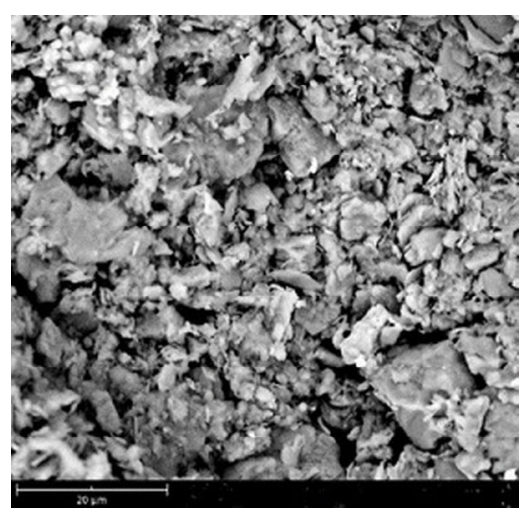

Fig. 7. Microstructure of sample S1 sintered at $1000^{\circ} \mathrm{C}$.

Sample C1 sintered at $1100^{\circ} \mathrm{C}$ exhibits a microstructure consisting of grains of dimension up to a few $\mu \mathrm{m}$ and agglomerates with dimensions about $20 \mu \mathrm{m}$ (Figure 5). The grains and agglomerates are distributed rather uniformly. Some of the grains have visible rounded edges but there are not observed the necks between grains that are characteristic 
for higher temperatures at which a greater amount of ash is present in slag form. Individual grains are weakly connected to each other. Numerous deep pores are also visible.

The microstructure of sample $\mathrm{S}$ is quite different (Figure 6). More homogeneous areas are adjacent to regions completely disordered. The main area of the sample is a solid material as closely interconnected grains by characteristic necks and with little or no pores [22]. Disordered regions are characterized by the existence of deep pores originating specific volume defects. In the whole sample can be seen also isolated single larger grains. These particular sintered grains are connected to each other by the necks which play a significant role in the diffusion paths for atoms and/or ions. In this way there are formed specific conduction channels.

Yet another microstructure is visible for sample S1 (Figure 7). It seems to be less homogeneous than sample $\mathrm{C} 1$ with a lower degree of agglomeration. More closely packed and larger individual grains are observed, with dimensions up to about $20 \mu \mathrm{m}$. Deep pores are also a characteristic feature of this microstructure.

\section{Conclusions}

On the basis of the results of studies it can be concluded that:

1. The value of dissipation factor $\tan \delta$ depends on both the kind of ash and the frequency range of the measurement. Frequency dependence of $\tan \delta$ is characteristic for the type of ash and the temperature at which the sample of ash was sintered. Therefore, the $\tan \delta$ measurements can be convenient tool for ash characterization.

2. In the case of ash obtained from pure coal (sample $\mathrm{C} 1$ ) dependence of $\tan \delta$ on frequency has a resonant character. For sintering temperature up to $800^{\circ} \mathrm{C}$ frequency of $\tan \delta$ maximum increases from $500 \mathrm{~Hz}$ to about $1 \mathrm{kHz}$ and the value of the maximum dissipation factor decreases. At $900^{\circ} \mathrm{C}$ it was observed a rapid increase of the maximum dissipation factor and slight slip to the lower frequency. Further sintering at temperatures 900,1000 and $1100^{\circ} \mathrm{C}$ leads to continuous decrease in the maximum dissipation factor value. It can be caused by the changes in microstructure, chemical transitions or similar effects.

3. For sample $\mathrm{S}$ obtained by the combustion of pure carbon with addition of biomass the relaxation character of $\tan \delta$ was observed for sintering temperatures in the range of 500$1000^{\circ} \mathrm{C}$. However, conducting character of $\tan \delta$ is observed for sample sintering at $1100^{\circ} \mathrm{C}$. This change may be associated with appearance of liquid slag phase.

4. For ash S1 resulting from the mixture of coal ash and kaolinite the conducting character of $\tan \delta$ are observed for samples sintered below $900^{\circ} \mathrm{C}$. Relaxation character of losses appear at $1000^{\circ} \mathrm{C}$. This change is complex to explain but is probably associated with the structure and composition transitions at high temperature for ash and kaolinite mixture.

5. The object of this work was to develop a method which could be used for laboratory studies of the sintering behaviour of ash samples as a direct and non-destructive test of slagging characteristics and slagging hazards assessment.

6. These preliminary studies are very promising. It seems that the direction of discussed research is appropriate and useful in practice. The $\tan \delta$ method for characterization of fuel ashes can be a very convenient and effective instrument. Nevertheless, the interpretation of the results is rather complicated and so this method still needs further work and more detailed analysis. 


\section{References}

1. D. Nowak-Woźny, W. Moroń, G. Hrycaj, W. Rybak, Archivum Combustionis 30 -3, 177-191 (2010)

2. J. Frenkel, J. Phys. (USSR) 9, 385-391 (1945)

3. E.J. Raask, Therm. Anal. 16, 91-102 (1979)

4. R.W. Bryers, Prog. Energy Combust. Sci. 22 (1), 29-120 (1996)

5. F.J. Frandsen, Energy Fuels 23, 3347-3378 (2009)

6. T.F. Wall, A. Lowe, L.J. Wibberley, I.McC. Stewart, Prog. Energy Combust. Sci. 5, 129 (1979)

7. A.Y. Al-Otoom, L.K. Elliott, T.F Wall, B. Moghtaderi, Energy Fuels 14 (5), 994-1001 (2000)

8. J. Tomeczek, H. Palugniok, Fuel 81 (10), 1251-1258 (2002)

9. D. Nutalapati, R. Gupta, B. Moghtaderi, T.F. Wall, Fuel Process. Technol. 88 (11-12), 1044-1052 (2007)

10. M. Zevenhoven-Onderwater, R. Backman, B.J. Skrifvars, M. Hupa, Fuel 80 (10), 1503-1512 (2001)

11. A.Y. Al-Otoom, G.W. Bryant, L.K. Elliott, B.J. Skrifvars, M. Hupa, T.F. Wall, Energy Fuels 14 (1), 227-233 (2000)

12. B.J. Skrifvars, R. Backman, M. Hupa, Fuel Process. Technol. 56, 55-67 (1998)

13. D. Lindberg, R. Backman, P. Chartrand, M. Hupa, Fuel Process. Technol. 105, 129$141(2013)$

14. J.C. van Dyck, M.J. Keyser, Fuel 116, 834-840 (2014)

15. T. Rizvi, P. Xing, M. Pourkashanian, L.I. Darvell, J.M. Jones, W. Nimmo, Fuel 141, 275284 (2015)

16. S. Chakravarty, A. Mohanty, A. Banerjee, R. Tripathy, G.K. Mandal, M. Raviathul Basariya, M. Sharma, Fuel 150, 96-101 (2015)

17. W.J. McCarter, G. Starrs, T.M. Chrisp, Cem. Concr. Res. 34, 1837-1843 (2004)

18. G.H. Zhang, K.C. Chou, Metall. Mater. Trans. B 43, 64-72 (2012)

19. D. Maurya, J. Kumar, Shripal, J. Phys. Chem. Solids 66, 1614-1620 (2005)

20. S. Hanjitsuwan, P. Chindaprasirt, K. Pimraksa, Int. J. Miner. Metall. Mater. 18-1, 94$99(2011)$

21. D.K.Mahato, A. Dutta, T.P.Sinha, Phys. B 406, 2703-2708 (2011)

22. W.D. Kingery, M. Berg, J. Appl. Phys. 26, 1205-1212 (1955) 